\title{
MALTRATO INFANTIL Y APROXIMACIONES MÉDICO - LEGALES DEL SÍNDROME DEL NIÑO SACUDIDO
}

\section{CHILD ABUSE AND THE MEDICAL - LEGAL APRROACH OF THE SHAKEN BABY SYNDROME}

Wendy Ramírez Alberto ${ }^{1}$

DOI: https://doi.org/10.5377//rd.v42i1.12921

\section{RESUMEN:}

En el presente artículo, se aborda el Síndrome del Niño Sacudido como una manifestación de las diversas formas de maltrato infantil, síndrome que presenta un cuadro clínico especifico por lo que se establecen algunas acotaciones médico - legales en torno al mismo.

PALABRAS CLAVES: medicina legal, violencia intrafamiliar, maltrato infantil, síndrome del niño sacudido, cuadro clínico, factores de riesgo.

\section{ABSTRACT:}

The Shaken Baby Syndrome (also known as Shaken Impact Syndrome) is one of the various forms of child abuse. Given the fact this syndrome has a very specific clinical presentation; this article encompasses several medical-legal aspects that must be considered when healthcare professionals are dealing with this type of child abuse.

KEYWORDS: legal medicine, domestic violence, child abuse, shaken baby syndrome, clinical presentation, risk fact.

Fecha de recepción: $12 / 10 / 2020$ Fecha de aprobación: 28/09/2021

1 Abogada y Fiscal del Ministerio Público de la República de Honduras, Egresada de la Maestría en Ciencias Penales de la Universidad de Costa Rica, Becaria del Sistema Alemán de Intercambio Académico (DAAD).

Correo Electrónico: wsramirez93@gmail.com 


\section{INTRODUCCIÓN}

El maltrato infantil es una de las manifestaciones de la violencia que causan mayor indignación y reproche social, sin embargo, aún nos encontramos muy lejos de conocer los datos reales respecto a la incidencia de este flagelo. Aunque no hay una respuesta única para indicar porque se desarrollan este tipo de maltratos en contra de la infancia, no es menos cierto que los estudios interdisciplinarios han logrado establecer una serie de factores de riesgo que son de común presencia en los casos de niñas y niños que han sido víctimas de malos tratos.

Dentro de estos factores de riesgo, y sobre todo en relación a maltratos de índole física, en muchas ocasiones y a través del abordaje médico se determina que los padres desconocen la afectación que pueden causar, por ejemplo, al ejercer disciplina con castigos físicos severos. En ese sentido, estudios históricos han concluido que dentro del entorno familiar, y en relación a la disciplina de los hijos e hijas, siempre se ha manifestado un lado oscuro que es propiciado más por la ignorancia que por la falta de amor ${ }^{2}$. Así, el maltrato infantil, como manifestación del abuso de la autoridad parental, en muchas culturas es parte de la ignorancia que los padres, madres o encargados que, ejerciendo su autoridad sobre la persona menor de edad, llegan incluso a causar lesiones físicas (externas, internas o ambas) y psicológicas.

La Organización Mundial de la Salud establece que el maltrato infantil, aparte de ser

2 Mario Alberto Víquez Jiménez, Maltrato físico en la niñez, un maltrato físico permitido. Estudio sobre autoridad parental, San José: Fondo de las Naciones Unidas para la Infancia, 2014, 35-53. un problema de considerable preocupación por las afectaciones graves que causa en el desarrollo de la población más joven, la incidencia de este tipo de malos tratos es desconocida por el ámbito de intimidad en el que generalmente se ejercen.

Con lo anterior, el modesto trabajo que se presenta trata de establecer las generalidades de este fenómeno de presencia mundial. Por eso, se ha tenido a bien plasmar el abordaje doctrinario y jurisprudencial desarrollado, con el propósito de brindan conceptos que orienten a comprender sobre qué debe entenderse como maltrato infantil; identificando a su vez la tipología de malos tratos, sus manifestaciones y secuelas.

Así mismo, el presente artículo se enfoca en una de las formas de maltrato infantil denominado Síndrome del Niño Sacudido. Este tipo de maltrato, tal como se menciona en líneas previas, en muchas ocasiones se ve propiciado por la ignorancia ya que el mismo se produce cuando la persona encargada (padre, madre, tutor, etc.) en un acto desesperado por calmar el llanto del niño o niña (principalmente en edades menores a los cuatro años) sacude de forma violenta su cuerpo provocándole, entre otras, lesiones cráneo-encefálicas que pueden llegar incluso a producir la muerte de la víctima.

Por lo anterior, es importante establecer algunas acotaciones médico - legales que deben considerarse en torno a este tipo de maltrato. En primer lugar, abordar el cuadro clínico que le permitirá al personal médico y de salud, advertir cuando se encuentren frente a una víctima menor de edad que sufre lesiones provocadas a través del mecanismo propio del Síndrome del Niño Sacudido. En segundo lugar, indicar cuál es el tipo de responsabilidad penal en que podrá incurrir el victimario dependiendo el tipo de lesión provocada. 
Finalmente, se brindan algunas recomendaciones para el personal médico y de salud respecto al abordaje de una persona menor de edad de la que se sospecha ha sido víctima de malos tratos y específicamente del Síndrome del Niño Sacudido, esto con el fin de salvaguardar la vida e integridad física del niño o niña, recordando el deber de denunciar este tipo de casos según lo establecido en la legislación interna.

\section{METODOLOGÍA}

La investigación que ha sido desarrollada es de carácter explicativo ${ }^{3}$ en virtud que se exponen e ilustran conceptos básicos referentes al tema de estudio que en este caso ha sido sobre el Síndrome del Niño Sacudido, presentando un enfoque meramente cualitativo ${ }^{4}$ ya que se parte de conceptos generales hacia aquellos de carácter específico, aportándose una explicación de cómo la medicina legal provee a la rama jurídica de una verdad científica que esclarece la senda investigativa en temas como la violencia intrafamiliar dentro de lo que se incluye el maltrato infantil. A su vez, empleándose para la presente investigación, fuentes primerias de información y en específico libros y artículos de revistas científicas ${ }^{5}$.

De todo lo anterior, y con base a la literatura y la doctrina consultada, la investigación concluye respecto que el maltrato infantil es un tema que reviste de mucha importancia para el debate académico y que su abordaje desde la rama jurídica resulta insuficiente. Representando de esta manera el personal médico y de salud un pilar

3 Rodrigo Barrantes Echavarría, Investigación: un camino al conocimiento, 2 ${ }^{\text {a }}$ ed. (San José: UNED, 2016), 86.

4 Ileana Ulate Soto y Elizarda Vargas Morúa, Metodología para elaborar una tesis, (San José: EUNED, 2016), 7-11. 5 Ibid., 74-75 importante para el diagnóstico de casos propios de maltrato infantil y en concreto del Síndrome del Niño Sacudido, lo que determina lo imperativo de un abordaje interdisciplinario de este síndrome.

\section{MEDICINA LEGAL Y EL DERECHO}

La literatura especializada coincide en que la Medicina Legal es el área de la ciencia médica que sirve como puente entre esta última y el derecho. Incluso, puede observarse una expansión en su función de colaboración con la rama jurídica, ya que la misma no se circunscribe necesariamente solo al ámbito legal civil y penal ${ }^{6}$.

Juan Antonio Sánchez y María Elena Albarrán la definen como "[...] aquella especialidad médica que aplicando sus conocimientos médicos y metodología accesoria complementa o auxilia a la administración de Justicia en todas sus jurisdicciones"

La medicina legal es así una ciencia auxiliar a la administración de justicia, encontrándose dentro de sus objetivos principales apoyar el esclarecimiento de los hechos objeto de litigio. Estos hechos, determinados a través del auxilio de la ciencia médica, pueden denominarse como una "verdad científica", ya que la medicina legal permitirá desarrollar un análisis especializado de las consecuencias jurídicas a través de la emisión de diagnósticos, permitiendo estos últimos orientar sobre todo al juzgador al momento de dictaminar sobre un caso en específico ${ }^{8}$.

6 Juan Antonio Sánchez y María Elena Albarrán, Medicina legal: concepto y definición. Historia. Importancia de la medicina

7 Ibíd.

8 Eduardo Vargas Alvarado, Medicina Legal, 4ta. Edición, México: Editorial Trillas, 2012, 15-16. 


\section{VIOLENCIA FAMILIAR Y MALTRATO INFANTIL}

Se ha mencionado que la medicina legal provee a la rama jurídica de una verdad científica que orienta de forma más clara, por decirlo de alguna manera, respecto a las consecuencias jurídicas sobre las cuales se deberá responder en torno a hechos concretos. En el tema que nos ocupa, la violencia intrafamiliar dentro de la que se incluye el maltrato infantil ${ }^{9}$, los aportes de la medicina legal estarán representados por la determinación de los indicadores de este tipo de violencia, especificación de la dinámica familiar y, en considerables ocasiones, permitirá el análisis de la credibilidad del testimonio de las víctimas que han enfrentado situaciones de violencia dentro de sus familias ${ }^{10}$.

La dinámica dentro del grupo familiar puede caracterizarse como compleja, por ende, con iguales tintes de complejidad, podrá presentarse el estudio o abordaje de toda actividad que se desarrolle en la intimidad de estos grupos sociales, por lo que los alcances de la ciencia jurídica resultan insuficientes, y es ahí donde la medicina legal toma un papel importante.

Así como la familia representa uno de los primeros grupos de socialización donde las hijas e hijos comienzan a aprender valores culturales, educativos, religiosos, entre otros, es dentro de los grupos familiares donde posiblemente tengan el primer encuentro con acciones violentas. A este tipo de violencia es a la que se le conoce como intrafamiliar o doméstica. La doctrina concuerda 9 Así en Gisbert Calabuig, Medicina Legal y Toxicología, 6ta. Edición, Editorial Masson, 2004, 486.

10 Ruth García Martin y Benito Morentin, Valoración forense integral del maltrato infantil intrafamiliar, Revista Española de Medicina Legal, Vol. 46, 2020, 28.

20-|Revista de Derecho. Vol. 42, No. 1, Año 2021 que, dentro de este tipo de violencia intrafamiliar se incluye la violencia de género y la generacional, es decir, violencia ejercida entre los miembros de la pareja, entre los hijos, o entre padres e hijos ${ }^{11}$.

La legislación nacional, específicamente en la Ley contra la Violencia Doméstica de Costa Rica, se establece un concepto que delimita los alcances de este tipo de violencia, indicándose que la misma comprenderá toda

Acción u omisión, directa o indirecta, ejercida contra un pariente por consanguinidad, afinidad o adopción hasta el tercer grado inclusive, por vínculo jurídico o de hecho o por una relación de guarda, tutela o curatela y que produzca como consecuencia, el menoscabo de su integridad física, sexual, psicológica o patrimonial. El vínculo por afinidad subsistirá aun cuando haya finalizado la relación que lo originó ${ }^{12}$.

Gisbert Calabuig menciona que lo actos de violencia en el ámbito familiar no es un fenómeno nuevo, y mucho menos de reciente estudio y abordaje. En ese sentido, la Organización Mundial de la Salud consciente de la prevalencia histórica de este tipo de violencia dentro del seno familiar ha manifestado que, si bien son considerables los esfuerzos por implementar políticas orientadas a prevenirla, no es menos cierto que siempre

11 Alvaro Capano y Ayrel Pachecho, Estrés Y Violencia Domestica: un estudio en adultos referentes de niños, niñas $y$ adolescentes, Prensa Medica Latinoamericana, Vol. VIII, 2014, 32.

12 Asamblea Legislativa de la República de Costa Rica, Norma No. 7586 - Ley contra la Violencia Doméstica, San José, abril de 1996. Consultada el 22 de septiembre de 2020 a través de la herramienta de búsqueda SINALEVI en la dirección electrónica http://www.pgrweb.go.cr/scij/ Busqueda/Normativa/Normas/nrm texto completo. aspx?nValor $1=1 \&$ nValor $2=27926$ 
se choca con esa barrera entre la vida privada y la esfera pública que frena el impacto de los esfuerzos preventivos y que pretenden reducir la incidencia de este tipo de situaciones dentro de los grupos familiares.

Aun así, este órgano internacional establece la importancia que deben tener los programas de prevención en torno a los factores desencadenantes de violencia en el ámbito familiar, siendo de principal preocupación la violencia ejercida en contra de las mujeres y la infancia ${ }^{13}$. En relación con esto, la Sala Constitucional de Costa Rica ha establecido la necesidad de desarrollar acciones preventivas en temas de maltrato infantil, indicando.

De igual manera el PANI deberá implementar programas de prevención del maltrato y abuso sexual infantil y ejercer el control y reducción de los factores de riesgo para evitar que esta violencia aparezca, se prolongue o cause daños mayores. No debe perderse de vista que el deber del PANI no es solo atender a las víctimas, sino prevenir la violencia y promover un ambiente saludable para el desarrollo de la niñez y la adolescencia. La acción preventiva promocional es necesaria y urgente porque debe evitarse que más niños, niñas y adolescentes sean expuestos a tratos crueles que vulneran sus derechos fundamentales $^{14}$ (La cursiva es del original).

13 Organización Mundial de la Salud, Prevención del Maltrato Infantil: qué hacer, y cómo obtener evidencias, Francia, 2009, 10-11.

14 Sala Constitucional de la República de Costa Rica, Resolución No. 15751 - 2008, del 21 de octubre del año 2008. Consultada a través de la herramienta de búsqueda Nexus el 29 de septiembre de 2020 en la dirección electrónica https:// nexuspj.poder-judicial.go.cr/document/sen-1-0007-421686

\section{1. ¿Qué es el maltrato infantil?}

La Convención de Derechos del Niño (CDN) establece en su artículo primero que, dentro del concepto de "niño" se comprenderá a toda persona menor de 18 años ${ }^{15}$, entendiéndose a su vez que en el concepto de infancia se incluye a toda persona que ostente una edad menor a la indicada, por consiguiente, en la definición de maltrato infantil proporcionada por este organismo se establece

El maltrato infantil se define como los abusos y la desatención de que son objeto los menores de 18 años, e incluye todos los tipos de maltrato físico o psicológico, abuso sexual, desatención, negligencia y explotación comercial o de otro tipo que causen o puedan causar un daño a la salud, desarrollo o dignidad del niño, o poner en peligro su supervivencia, en el contexto de una relación de responsabilidad, confianza o poder. La exposición a la violencia de pareja también se incluye a veces entre las formas de maltrato infantil ${ }^{16}$.

A su vez, en su artículo 19, la CDN orienta a los Estados parte a tomar todas las medidas que sean necesarias para evitar cualquier tipo de maltrato, agresión o afectación a los niños, niñas $\mathrm{y}$ adolescentes, indicando en ese sentido

15 Organización de las Naciones Unidas, Convención sobre los Derechos del Niño, Resolución 44/25, 1989. Consultada a través de la página electrónica de la ONU a través de la dirección https://www.ohchr.org/SP/ProfessionalInterest/ Pages/CRC.aspx

16 Obtenido a través de Organización Mundial de la Salud, Temas de Salud: maltrato de menores, consultado el 10 de septiembre de 2020, a través de la dirección https://www.who. int/topics/child abuse/es/ 
Los Estados Partes adoptarán todas las medidas legislativas, administrativas, sociales y educativas apropiadas para proteger al niño contra toda forma de perjuicio o abuso físico o mental, descuido o trato negligente, malos tratos o explotación, incluido el abuso sexual, mientras el niño se encuentre bajo la custodia de los padres, de un representante legal o de cualquier otra persona que lo tenga a su cargo ${ }^{17}$.

La conclusión que se desprende de los dos conceptos anteriores es la relación directa que se establece entre una figura de autoridad, custodia o cuido, y el maltrato ejercido en contra de la persona menor de edad. Es decir, tanto la Organización Mundial de la Salud, así como las disposiciones contenidas en la Convención de Derechos del Niño refieren que, en primera instancia, pero no exclusivamente, los maltratos propiciados contra niños y niñas se originan dentro del ámbito familiar, lo que les coloca en una posición considerablemente vulnerable y de mayor riesgo frente a este tipo de violencia.

El Tribunal de Familia del sistema judicial de Costa Rica a través de Resolución 003412001 ilustra en igual sentido esta relación entre una figura de autoridad y el origen del maltrato infantil, manifestando

La agresión intrafamiliar contra los menores de edad, es producida por una acción u omisión de la persona encargada del menor o sus padres, y que le causa un daño físico o psicológico. Esta agresión es crónica, permanente o periódica. Además, esta agresión se produce por una combinación de condiciones y factores determinantes y que

17 Convención sobre los Derechos del Niño, resolución 44/25, 20 de noviembre de 1989. disponen el control de la persona más fuerte y poderosa (padre o madre) sobre su hijo (el subrayado no es del original $)^{18}$.

Ruth García Martín y Benito Morentin establecen un concepto de maltrato infantil a través del cual dilucidan que este tipo de acciones violentas en contra de la infancia se desarrollan principalmente dentro del hogar, indicando

El maltrato infantil intrafamiliar (MII) se puede definir como toda acción, omisión o trato negligente, no accidental, que priva al/a la menor de sus derechos y su bienestar, que amenaza o interfiere en su desarrollo físico, psíquico o social y cuyos autores son personas del ámbito familiar. La negligencia es el tipo más frecuente, seguido del maltrato emocional y el físico. Los casos de abuso sexual representan una minoría ${ }^{19}$.

Como puede observarse a través de los conceptos aportados, la necesidad de implementar medidas preventivas y una legislación especializada, y sobre todo apoyada por disciplinas diferentes a la jurídica, que permitan un abordaje correcto de las víctimas de maltrato infantil, es en miras de reducir la incidencia de este tipo de violencia sobre todo por la condición de vulnerabilidad de las víctimas que no pueden denunciar por sí mismas, lo que lleva a afirmar que la cifra oscura de este tipo de acciones es considerable.

18 Tribunal de Familia, Resolución No. 00341-2001, del 05 de marzo de 2001. Consultada a través de la herramienta de búsqueda Nexus el 27 de septiembre de 2020 en la dirección electrónica https://nexuspj.poder-judicial.go.cr/document/sen$\underline{1-0034-144875}$

19 García Martín y Morentin, 29. 


\subsection{Tipos de maltrato infantil}

Calabuig proporciona la siguiente clasificación de los malos tratos ejercidos en contra de la infancia, clasificación a la que se apega la doctrina mayoritaria

1. Por omisión:

a. carencias físicas: se incluyen la falta de suministro de alimentos, medicación adecuada, protección contra cambios drásticos de clima, dietas, entre otros.

b. carencias afectivas: en igual de importancia que las carencias físicas porque producen, en entre otras, consecuencias como hiperactividad, intentos de suicidio, baja autoestima, trastornos de conducta, etc.

2. Por acción:

a. Maltrato físico: presencia de contusiones en el cuerpo del niño o niña que han sido provocados ya sea por empujones, pateamientos, zarandeo. Se incluyen a su vez heridas por instrumentos punzantes, cortantes, quemaduras por líquidos, solidos calientes, entre otros.

b. Abuso sexual: en el caso de Costa Rica, para el año 2019 se presentó como una de las causas de violencia intrafamiliar más común ${ }^{20}$. Este es considerado un problema de salud pública.

20 Stephanie Cohen Rosentock y Esteban Cob Guillén, Abuso sexual en el paciente pediátrico, Revista de Medicina Legal de Costa Rica, Vol. 36, 2019, 54. c. Maltrato psíquico: dentro de los que se enmarcan los castigos excesivos, amenazas graves, coacción, encierros prolongados, inter alia ${ }^{21}$.

Las graves consecuencias que traen aparejadas, cualquiera de los tipos de violencia o malos tratos, no se discute. Incluso, se menciona que habrá una especial afectación en el desarrollo de las víctimas menores de edad por su condición de vulnerabilidad y los efectos imborrables de la victimización debido a su nivel de dependencia y falta de autonomía frente a la población adulta $^{2223}$. A su vez, la Organización Mundial de la Salud indica que "más allá de sus consecuencias sanitarias y sociales, el maltrato infantil tiene un impacto económico que abarca los costos de la hospitalización, de los tratamientos por motivos de salud mental, de los servicios sociales para la infancia y los costos sanitarios a largo plazo"24.

Las estadísticas oficiales presentadas por el Observatorio de violencia de género contra las mujeres y acceso a la justicia del Poder Judicial de Costa Rica, correspondientes al año 2019, son concordantes con la literatura especializada ya que se habla de un predominio del ejercicio de la violencia en perjuicio de niños ${ }^{25}$, es decir, el porcentaje de niñas víctimas de violencia doméstica tiende a ser menor, tal como se observa en el siguiente gráfico.

21 Calabuig, 489.

22 García Martín y Benito Morentin, 29.

23 Algunas de las consecuencias que se observan en adultos que sufrieron malos tratos durante la infancia se encuentran: consecuencias en la conducta y la salud mental, el maltrato puede contribuir a las enfermedades del corazón, al cáncer, al suicidio y a las infecciones de transmisión sexual.

24 Organización Mundial de la Salud, Maltrato Infantil: datos y cifras. Consultada el 23 de septiembre de 2020 en la dirección electrónica https://www.who.int/es/news-room/factsheets/detail/child-maltreatment

25 Calabuig, 489.

Revista de Derecho. Vol. 42, No. 1, Año 2021 -23 


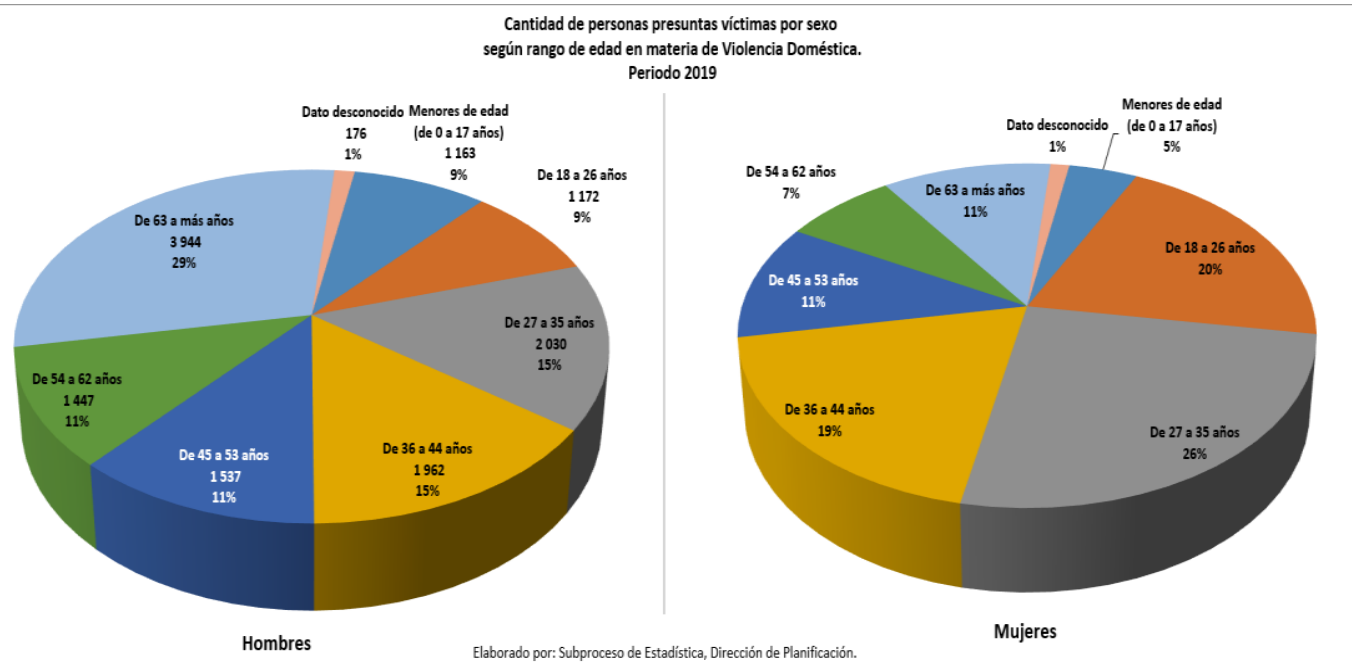

Fuente: Observatorio de violencia de género contra las mujeres y acceso a la justicia ${ }^{26}$.

\section{PERSONA MENOR EDAD VÍCTIMA DE MALTRATO.}

Un niño o niña maltratados presentarán afectaciones significativas en su salud, afectaciones que pueden ser por traumas físicos, psicológicos, emocionales y que vienen acompañados de señales de alarma como ser el absentismo escolar, defectos de socialización e higiene, aptitud apática, temerosa, triste, o con una actitud a la defensiva, inter alia ${ }^{27}$.

Concordantes con lo anterior son María Elena Albarrán y José Antonio Sánchez, que citando a Mediuris, proporcionan una serie de indicadores para establecer que la persona menor de edad ha sufrido agresiones o ha sido víctima de malos

26 Se pueden consultar más datos estadísticos relacionados con violencia doméstica en la dirección electrónica del Observatorio de violencia de genero https://observatoriodegenero. poder-judicial.go.cr/index.php/soy-especialista-y-busco/estadisticas/delitos-sexuales

27 Calabuig, 490.

24-|Revista de Derecho. Vol. 42, No. 1, Año 2021 tratos, enumerando los siguientes: 1) Lesiones múltiples y variadas en estadíos diferentes (ej: hematomas, fracturas); 2) Sitios donde las lesiones no son habituales o mecanismos de producción extraños o sospechosos (fractura por retorcimiento); 3) Frecuentar constantemente los servicios médicos o de emergencia; 4) Retrasos del crecimiento y/o del aprendizaje; 5) Falta cuidados (ej: vestiduras, inmunizaciones, ropas, higiene, etc. $)^{28}$

\subsection{Sobre las lesiones.}

Dentro de las principales afectaciones físicas que permitirán advertir cuándo una persona menor ha sido o está siendo víctima de malos tratos son las lesiones. Sin embargo, su identificación no siempre resulta sencilla, sobre todo cuando se encuentran en partes del cuerpo poco visibles, por eso se recomienda poner especial énfasis en la exploración ya que algunas lesiones físicas serán más sutiles que otras y según el tipo de maltrato del que se trate serán de naturaleza distinta.

28 Ibíd., 95. 


\section{i) Lesiones por abandono}

Pueden incluirse aquellas tanto en el aspecto físico como mental, es decir, la persona menor de edad presenta deficiencias en el ámbito escolar, presenta una mala alimentación, vestuario descuidado, falta de atención sanitaria que puede observarse en su dentadura, lesiones no atendidas en su momento, falta de demostración afectiva $\mathrm{y}$ protección, etcétera ${ }^{29}$.

\section{ii) Lesiones físicas}

Respecto a este tipo de lesiones debe ponerse mucha atención a la historia que los padres o encargados de la persona menor de edad brinden ante el médico ya que en muchas ocasiones no se encuentra relación entre el traumatismo y el mecanismo con el que se provocó la lesión ${ }^{30}$.

Particularmente en el caso de Costa Rica, a través de Ley No. 8654 se adicionó al Capítulo II del Código de la Niñez y la Adolescencia (artículo 24 bis) el derecho de los niños, niñas y adolescentes a ser disciplinados sin castigo físico ni trato humillante por parte de la madre, padre, encargados de la guarda y crianza, incluso, extendiéndose esta prohibición al personal de centros educativos, de salud, inter alia.

La jurisprudencia del Tribunal de Familia ha amparado este derecho en mención, indicando

Este tribunal no desconoce la existencia jurídica del derecho de corrección que los padres tienen para con sus hijos en virtud de aquel contenido personal de los llamados derechos de la Autoridad Parental, que

29 Ibíd., 96.

30 Édgar Alonso Madrigal, Aspectos medico legales del síndrome del menor agredido, Acta Medica Costarricense, Vol. $52(4), 2010,205$. complementan los otos dos contenidos de representaciónydeadministración delosbienes [...] Las modernas corrientes de la psicología actual hacen que se tenga que el castigo de tipo físico se vea hoy como una solución no solo obsoleta, sino fundamentalmente como una forma de actuar que necesariamente se revierte en una concatenación de hechos mas conflictivos en la relación con los hijos; por cuanto desencadena precisamente en hechos de violencia que sin duda marcará a los menores de edad hacia su desarrollo y dejará que ello provoque un estigma hacia las nuevas generaciones; pero además porque el castigo físico denota la falta de autoridad que en su momento no se ejerció con los niños y que ahora se ve como una finalidad no de corrección, sino de maltrato y castigo para conductas previsibles, de necesarios diálogo y comunicación y con existencia de otro tipo de correcciones dentro del hogar. $-^{31}$

Calabuig y Albarrán Sánchez indican que las lesiones pueden ser de distinta naturaleza y pueden consistir en

a) Cutaneomocosas: 1) Equimosis y hematomas (principalmente en la cabeza); 2) Heridas: contusas o por arma blanca; 3) Quemaduras: pueden complicarse por descuido o negligencia.

b) Esqueléticas: 1) Fracturas en la cabeza: cráneo, hemorragias y trastornos neurológicos que pueden comprometer la vida de la persona menor de edad; 2) Fracturas en extremidades: arrancamientos metafísicos; 3) Fracturas de costillas; 4) La

31 Tribunal de Familia, Resolución No. 01624-2007 del 20 de noviembre de 2007. Consultada el 07 de octubre de 2020 a través de la herramienta de búsqueda Nexus en la dirección electrónica https://nexuspj.poder-judicial.go.cr/document/sen1-0034-393051

Revista de Derecho. Vol. 42, No. 1, Año 2021 $\mid-25$ 
pelvis: producida por traumatismos muy violentos.

c) Viscelares: 1) Hematoma subdural: entraña compresión cerebral y trastornos neurológicos; 2) Lesiones abdominales: rupturas de intestino, hígado y bazo son poco comunes, pero pueden conducir a la muerte; 3) Lesiones genitales: de variada índole dependiendo si la víctima es niña o niño.

Dependiendo de la forma en que se produzcan las agresiones, estas pueden ser por un hecho agudo o por un periodo prolongado por lo que el médico debe tener la capacidad y el conocimiento necesario para determinar, y con significativo cuidado, cuánto tiempo de evolución puede presentar una lesión ${ }^{32}$.

Dependiendo del objeto, la intensidad de la violencia, la intensión, si han sido varias lesiones o los mecanismos especiales con los que se provocaron, hay cuadros clínicos específicos que permiten identificar y determinar algunos síndromes de agresión, como ser el Síndrome de Munchausen por poder ${ }^{33}$, Síndrome de suspensión del niño por los brazos ${ }^{34}$ y el Síndrome del Niño Sacudido ${ }^{35}$.

Este último, el Síndrome del Niño Sacudido, que desarrollaremos en el siguiente apartado, se caracteriza por ser una forma de abuso físico

32 Madrigal, 205.

33 Solicitud de ayuda reiterada o de diagnóstico para la persona menor de edad ante síntomas que suelen ser variados (convulsiones, cefaleas, fiebre, etc.) que pueden ser provocadas por la administración de medicamentos para que las mismas se produzcan.

34 Consiste en lesiones no graves pero que puede provocar arrancamientos ligamentosos, desgarros musculares 0 elongación del plexo braquial.

35 Tipo de maltrato infantil físico provocado en personas menores de edad, principalmente en edad lactante y hasta los cuatro años. determinado por algunos hallazgos clínicos sobre todo en niños y niñas que son abordados en salas de emergencia en centros hospitalarios.

\section{SÍNDROME DEL NIÑO SACUDIDO. APROXIMACIONES MÉDICO LEGALES}

Las formas de malos tratos o violencia ejercida en contra de la infancia pueden ser de diversa índole. Un tipo de maltrato infantil de índole física que, según los expertos, presenta un abordaje un tanto complejo es el denominado Síndrome del Niño Sacudido mismo que es considerado como un problema de salud pública de consecuencias considerables y que resulta de una compleja interacción de los factores de riesgo del individuo, la familia y la sociedad.

El diagnóstico de este síndrome puede requerir de cierta experticia, rigurosidad y colaboración entre expertos, sobre todo del personal médico, al momento de evaluar a una persona menor de edad de la que se sospecha ha sido víctima de malos tratos, por lo que es importante establecer algunos síntomas que determinan el cuadro clínico de este tipo de violencia.

Las lesiones provocadas a la persona menor de edad pueden enmarcarse como no accidentales ya que, aunque la persona encargada no conozca los efectos nocivos para la salud del niño o niña, en la mayoría de ocasiones ha sido por negligencia del padre, madre o encargado que tratando de parar el llanto de la víctima sacuden violentamente su cuerpo, lo que provoca serias lesiones internas.

Este tipo de lesiones se producirán al sacudir de forma violenta a la persona menor de edad, tomándola del tórax o las extremidades, sacudiéndole de adelante hacia atrás. Al niño o niña se le sacude de forma tan fuerte que el cerebro rebota dentro del cráneo y, a través de 
un mecanismo denominado de aceleración desaceleración de la cabeza, se produce la lesión de las venas generando sangrado en el cerebro y en los nervios ópticos ${ }^{36}$.

Es un síndrome que se presenta principalmente en personas menores de dos años ya que el tamaño de la cabeza es desproporcionado con respecto al resto del cuerpo propiciando el desarrollo de lesiones internas en el cerebro, no encontrándose en muchos de los casos signos externos de violencia ${ }^{37}$. Lo susceptible del cerebro de la persona menor de edad reside en que el mismo no está desarrollado en totalidad y sus áreas no funcionan de forma completa, por eso este tipo de lesiones pueden provocar secuelas motoras, visuales, del lenguaje o del aprendizaje. A su vez, el daño cerebral dependerá de la fuerza y la duración de la sacudida ${ }^{38}$.

\subsection{Cuadro clínico del Síndrome del Niño Sacudido}

La Real Academia Española define un "cuadro clínico" como el "conjunto de síntomas que presenta una persona enferma o que hacen característica una enfermedad" ${ }^{39}$, el síndrome del niño sacudido estará caracterizado principalmente

36 Ana Teresa Alvarado Guevara y Karen Garro Vargas, El síndrome del niño sacudido, Revista de Medicina Legal de Costa Rica, Vol. 25, 2008, 37-42.

37 Calabuig, 491.

38 Pilar Tejada Palacios, Noemí Núñez Enamorado y Berta Zamora Crespo indican que esta forma de maltrato es la causa de muerte $\mathrm{y} / \mathrm{o}$ secuelas neurológicas graves por maltrato, siendo a su vez la causa más frecuentes de muerte no natural en la infancia. Así en Manifestaciones oftalmológicas del niño maltratado. Traumatismo craneal no accidental, Boletín de la Sociedad Oftalmológica de Madrid, No. 54 (2), 2014. Consultada a través de la dirección electrónica https:// sociedadoftalmologicademadrid.com/revistas/revista-2014/ m2014-03.html

39 Diccionario de la Real Academia Española. Consultado el 20 de septiembre de 2020, a través de la dirección electrónica https://dle.rae.es/cuadro por los siguientes hallazgos clínicos:

- Hematoma subdural u subaracnoideo ${ }^{40}$.

- Edema cerebral difuso ${ }^{41}$

- Hemorragias retinianas, que en el $80 \%$ de los casos están presentes ${ }^{42}$.

Identificar este síndrome constituye un verdadero reto para las y los profesionales de salud ya que la historia clínica recogida es muchas veces inexacta, engañosa, los hallazgos del examen físico se presentan con datos inespecíficos y los médicos, en su mayoría, no lo incluyen en el diagnóstico diferencial ${ }^{43}$. Incluso, se afirma que este tipo de maltrato infantil es la causa más frecuente de traumatismos cráneo-encefálicos graves en el lactante antes del año de edad ${ }^{44}$.

Como se indicaba previamente, la presencia de lesiones como hematomas subdurales $\mathrm{y}$ hemorragias retinianas serán de importante relevancia para determinar una agresión en contra de la persona menor de edad a través

40 Acumulación de sangre entre la cubierta del cerebro (duramadre) y la superficie del cerebro. Consultado así en la Biblioteca Nacional Virtual de Medicina de los Estados Unidos el 24 de septiembre de 2020 a través de la dirección electrónica https://medlineplus.gov/spanish/ency/article/000713.htm

41 Sobre los traumatismos cerebrales se indica que aproximadamente el 20 por ciento de ellos está relacionado con la violencia tal como sucede en el maltrato infantil. Para mayor referencia abordar "Traumatismo Cerebral: Esperanza en la investigación”, en la dirección electrónica https://espanol.ninds. nih.gov/trastornos/el traumatismo cerebral.htm?css=print

42 M. Rufo Campos, El síndrome del niño sacudido, Cuadernos de Medicina Forense, Vol. 12, 2006, 40.

43 El diagnóstico diferencial es utilizado para distinguir entre enfermedades físicas o mentales que causa síntomas parecidos. Este puede tomar en cuenta todas las enfermedades que podría estar causando los síntomas. Consultado así en la Biblioteca Nacional Virtual de Medicina de los Estados Unidos, el 24 de septiembre de 2020 a través de la dirección electrónica https://medlineplus.gov/lab-tests/differential-diagn osis/?fbclid=IwAR0mINXVRm35xxqdAenf4NZIkpb7JU6xja hDLFsbLRWs83CSS1LcOsBKhik

44 Rufo Campos, 40. 
del mecanismo de zarandeo, sin embargo, es importante a su vez establecer algunas de las secuelas que podrían presentarse y así afectar la salud de la víctima.

\section{Secuelas neurológicas por daño cerebral o craneal}

J. Giménez Pando, E. Pérez Arjona y M. Dujovny y F. G. Díaz establecen que este tipo de secuelas pueden dividirse en cuatro grupos.

El primer grupo, secuelas motoras, dentro de las que pueden presentarse retraso mental más o menos marcado y/o déficit neurológico y comportamientos autistas sin poder pronunciar palabras de forma fluida. Autores como Alvarado Guevara, Garro Vargas y Giménez Pando indican que el pronóstico de las secuelas a largo plazo en la mayoría de casos resulta ser muy pobre, por lo que para apreciar los cambios estructurales deberá apoyarse de estudios de Resonancia Magnética, y para la determinación de cambios funcionales resultaran más útiles estudios nucleares ${ }^{45}$.

Un segundo grupo de secuelas, Secuelas visuales, caracterizadas principalmente por las hemorragias retinianas que, aunque no producen secuelas considerables, existe una correlación importante entre su extensión y el grado daño neurológico debido a la coexistencia de lesiones cerebrales ${ }^{46}$. La presencia de este tipo de secuelas habitualmente indica un zarandeo violento ${ }^{47} 48$.

45 J. Giménez Pando, E. Pérez Arjona y otros, Secuelas neurológicas del maltrato infantil, revisión bibliográfica, Sociedad Española de Neurocirugía, Vol. 18 (2), 2007, 97.

46 Ibíd., 97-98

47 Tejada Palacios y otros

48 Ma. Eliana Eberhard, Georgette Pose L. y otros, Maltrato infantil, TEC y diagnóstico por imágenes, Revista Chilena de Pediatría, Vol. 82 (3), 2011, 234. Sobre la evaluación oftalmológica para detección de hemorragia retiniana la misma resulta de vital importancia en casos como estos. La severidad que se observe en la hemorragia retineal estará completamente relacionada con la severidad de la injuria
Las secuelas del lenguaje y aprendizaje, pueden presentarse diversos trastornos o anomalías en el lenguaje (disfasias mixtas) que son causa de lesiones cerebrales. Pueden ser de índole variada puramente motoras o sensitiva, aunque a su vez puede existir un retraso en el aprendizaje cuando se dañan otros órganos como el oído o la vista ${ }^{49}$.

Un último tipo de secuelas comprende el retraso mental y alteraciones de la memoria. El retraso mental se presenta con mayor frecuencia en niños que han sido maltratados físicamente que en aquellos que han sufrido traumatismos accidentales, lo que conlleva a firmar que el retraso mental constituye una causa etiológica de retraso mental ${ }^{50}$.

Es importante también mencionar que las lesiones espinales y medulares en víctimas de maltrato infantil se presentan de forma poco frecuente en comparación a las lesiones cerebrales $^{51}$.

La jurisprudencia nacional reconocer la dificultad de identificar este tipo de lesiones $y$, mayor medida, obtener prueba directa que establezca que efectivamente se ha sacudido de forma violenta el cuerpo de la persona menor de edad, así la Sala Tercera de la Corte Suprema de Costa Rica a través de la Resolución no. 007162012, ha manifestado

[...] en ninguna forma viene a desvirtuar el hecho evidente -a partir de los hallazgos de la autopsia- que aquél (víctima) en al menos dos oportunidades sufrió agresiones físicas por parte de sus progenitores o cuidadores, las

intracraneal que pudo haber sufrido el menor durante la sacudida.

49 Giménez Pando y otros, 98.

50 Ibíd

51 Ibíd 
cuales incluso se prolongaron hasta el día en que es llevado de emergencia a la clínica más cercana [...] Sobre la prueba en general en este tipo de delitos, la jurisprudencia de esta Sala Tercera, ha indicado: “...en este tipo de casos (síndrome de niño agredido) la prueba resulta escasa, pues se trata de abusos físicos perpetrados en el seno familiar, donde los agresores tienen a buen recaudo el exponerse a la mirada de terceros. Aunado a lo anterior, la situación se complica porque el delito no se consuma a partir de un acto preciso y determinado, sino que ello se consigue a consecuencia de una serie de acciones que se van ejecutando y prolongando en cierto espacio de tiempo, todas las cuales pueden progresivamente ir dejando lesiones y secuelas que al final de cuentas contribuyen (unas en mayor grado que otras) a la producción del resultado..." (la cursiva es del original) $)^{52}$.

Ilustrativo sobre los síntomas, alcances y el proceso de abordaje de una persona menor de edad de la que se sospecha ha sido objeto de agresión o maltrato infantil, es el extracto que, de los diagnósticos médicos, refiere la Resolución 00591-2017 del Tribunal de Apelación de Sentencia Penal del Segundo Circuito Judicial de San José, en la que se indica

Según se consignó en el expediente médico del HNN, el 09/04/12 a la 1.53 pm, desde el ingreso de la niña al Servicio de Emergencias se sospechó que había sido víctima de agresión, puesto que presentaba bradicardia y apnea $y$ status convulsivo. Posteriormente ese mismo día, se consigna como resultado de la interconsulta de Oftalmología que el fondo de ojo realizado

52 Sala Tercera de la Corte Suprema de Costa Rica, Resolución No. 000716-2012 de los 20 días del mes de abril del año 2012. Consultada a través de la herramienta de búsqueda Nexus el 20 de septiembre de 2020 en la dirección electrónica https:// nexuspj.poder-judicial.go.cr/document/sen-1-0006-781664 en ambos ojos arroja múltiples hemorragias intraretinianas puntiformes, indicándose que la paciente presenta datos sugestivos de SNA (Síndrome del Niño Agredido) por los hallazgos oftalmológicos. Luego, al ser las 2.26 pm de ese mismo día, el Dr. Calzada de Emergencias, consigna que se le realiza TAC como sospecha de Síndrome del Niño Agredido por múltiples equimosis, se conversa con radiología a quien les impresiona hemorragia subaracnoidea y el Fondo de ojo realizado indica la presencia de hemorragias retinianas bilaterales. Una vez en la UCI, el Dr. Guzmán Haeussler, quien declaró en debate, consignó en el expediente médico de [Nombre 002], ese mismo día de su ingreso, que la niña se mantenía en observación por Síndrome del Niño Agredido, por presentar Hemorragia subaracnoidea, hemorragias retinianas y status convulsivo, síntomas, según los médicos que declararon en debate, altamente sugestivos de ese Síndrome, ya bien conocido a nivel clínico por los médicos pediatras del Hospital Nacional de Niños (la cursiva y resaltado son del original $)^{53}$.

En un estudio desarrollado en el departamento de cuidados intensivos de Hospital Universitario Niño de Jesús en Madrid España, la mayoría de pacientes que ingresaron a la Unidad de Cuidados Intensivos Pediátricos se encontraban en edad lactante e ingresaban por alteraciones neurológicas, observándose lesiones en las pruebas de imagen cerebral lo que se corresponde al síndrome del niño sacudido, considerándosele la forma de maltrato más frecuente en esa edad ${ }^{54}$.

53 Tribunal de Apelación de Sentencia Penal del Segundo Circuito Judicial de San José, Resolución No. 00591-2017, del 19 de mayo de 2017. Consultada a través de la herramienta de búsqueda Nexus el 20 de septiembre de 2020 a través de la dirección electrónica https://nexuspj.poder-judicial.go.cr/ document/sen-1-0034-719509

54 A. Ríos, J. Casado Flores y otros, Maltrato infantil grave en la unidad de cuidados intensivos pediátricos, Anales de 
Lo complejo del abordaje de este tipo de maltrato físico es que los síntomas suelen ser inespecíficos presentándose como los menos graves la irritabilidad, temblores o vómito; sin embargo, se recurre al apoyo médico hasta que los mismos se vuelven intensos como en el caso de convulsiones, dificultad respiratoria, apnea, bradicardia o coma profundo ${ }^{55}$. Como se ha indicado, en sala de urgencias cuando el papá, mamá o encargado de la persona menor de edad acuden a la asistencia médica porque se ha complicado la salud del niño o niña luego de haber sido sacudido violentamente, no proporcionan una historia coherente.

Ante esta situación, las doctoras Ana Teresa Alvarado Guevara y Karen Garro Vargas indican algunas actividades en las que se excusan las personas encargadas, justificando que a través de las mismas se provocaron las lesiones propias del Síndrome del Niño Sacudido, cuando en realidad no ha sido así. Actividades que no causan las mismas lesiones: 1) La caída del bebé de un mueble o coche; 2) Cuando se lo hace rebotar o cabalgar en las rodillas de un adulto; 3) Si la persona a cargo del cuidado del bebé lo lleva en una mochila o cargador mientras trota o corre; 4) Lanzar al bebé al aire y atraparlo; 5) Una sacudida en el asiento del automóvil cuando el conductor frena repentinamente ${ }^{56}$.

Sobre la no concordancia entre la historia que los padres o encargados aportan en el centro hospitalario y el diagnóstico médico que se proporciona luego de evaluarse a la persona $\underline{\text { menor edad es ejemplificativa la Resolución }}$ Pediatría, Vol. 71 (1), 2009, 66.

55 Ibíd., 67.

56 Ana Teresa Alvarado Guevara y Karen Garro Vargas, El síndrome del niño sacudido, Revista de Medicina Legal de Costa Rica, Vol. 25, 2008, 40.
No. 00335-2013 del Tribunal de Apelación de Sentencia Penal de Guanacaste en la que se indica

La relevancia jurídico penal de la deplorable condición de salud en que se lleva a la bebé al hospital, surge desde el mismo momento en que, al ser recibida en el nosocomio, se le diagnostica de entrada, síndrome de niña agredida [...] la reacción del encartado frente a ese hecho, desde un inicio, ante personal médico e incluso en el plenario, fue la de dar falsas explicaciones sobre las causas de las graves lesiones propias del síndrome de niña sacudida que presentaba la bebé y que de ninguna manera pudieron ser sustentadas con esa explicaciones, por ser incompatibles con la explicación médica del $\operatorname{caso}^{57}$.

A su vez, es importante mencionar que los médicos especialistas concuerdan que, dependiendo la fuerza con que se sacuda al niño o niña, podría inclusive ocasionársele fracturas costales diversas ${ }^{58}$, sin embargo, la ausencia de las mismas no debe hacer que se descarte que se ha maltratado al menor de edad a través del mecanismo de zarandeo. El Tribunal de Apelación de Sentencia Penal, abordando un caso de maltrato infantil, estableció sobre este punto en específico lo siguiente

También apunta el impugnante que, si hubiese sido un caso de niño agredido, la menor de edad hubiese mostrado, por ejemplo, lesiones en las vértebras cervicales o bien, fracturas antiguas ya consolidadas. Sobre esto, basta señalar que lo primero (presencia

57 Tribunal de Apelación de Sentencia Penal de Guanacaste, Resolución No. 00335-2013 del 17 de diciembre de 2013. Consultada a través de la herramienta de búsqueda Nexus el 29 de septiembre de 2020, en la dirección electrónica https:// nexuspi.poder-judicial.go.cr/document/sen-1-0034-595547 58 Alvarado Guevara y Garros Vargas, 38-39. 
de lesiones en las vértebras cervicales) no solo no resulta indispensable, sino que, por el contrario, lo común es la ausencia de fracturas $\mathrm{u}$ otros signos externos de violencia. $\mathrm{Y}$ es que las hemorragias retinianas y los hematomas subaracnoideos se dan porque el tamaño y el peso de la cabeza de la víctima resultan desproporcionados con respecto al resto del cuerpo, de ahí que la sacudida o el zarandeo provoca que el cerebro oscile e impacte con el cráneo, rompiéndose los vasos sanguíneos ${ }^{59}$.

\subsection{Factores de riesgo}

Aunque las acciones $u$ omisiones constitutivas de maltrato infantil pueden verse propiciadas por múltiples factores (sociales, económicos, culturales, etc.), en forma general, los expertos coinciden en que los siguientes agentes han sido consistentes en casos de maltrato infantil

\begin{tabular}{|c|c|}
\hline \multicolumn{2}{|c|}{$\begin{array}{l}\text { Factores de riesgo del maltrato infantil } \\
\text { (aplicable al Síndrome del Niño Sacudido) }\end{array}$} \\
\hline Factores del niño & $\begin{array}{l}\text { - } \quad \text { Menores de } 4 \text { años. } \\
\text { No haberse planeado la } \\
\text { concepción del bebé. } \\
\text { Requerir de mayor atención } \\
\text { y cuidado por condiciones } \\
\text { especiales, irritabilidad, llorar } \\
\text { mucho o presentar rasgos físicos } \\
\text { anormales. }\end{array}$ \\
\hline $\begin{array}{l}\text { Factores de } \\
\text { los padres o } \\
\text { cuidadores }\end{array}$ & $\begin{array}{l}\text { Antecedentes personales de } \\
\text { maltrato infantil }{ }^{60} \text {. } \\
\text { No hacerse cargo de cuidar al } \\
\text { niño/niña. } \\
\text { - Consumo indebido de alcohol } \\
\text { o drogas. } \\
\text { - Dificultades económicas. }\end{array}$ \\
\hline
\end{tabular}

59 Tribunal de Apelación de Sentencia Penal - Segundo Circuito Judicial de San José, Resolución 00591 - 2017 de los 19 días del mes de mayo del año 2017. Consultada a través de la herramienta de búsqueda Nexus el 29 de septiembre de 2020, en la dirección electrónica https://nexuspj.poderjudicial.go.cr/document/sen-1-0034-719509

60 Un estudio ejemplificativo sobre este tipo de riesgo en

\begin{tabular}{|l|ll|}
\hline $\begin{array}{l}\text { Factores sociales } \\
\text { y comunitarios }\end{array}$ & $\bullet$ & $\begin{array}{l}\text { Desigualdades sociales y de } \\
\text { género. } \\
\text { Niveles elevados de desempleo } \\
\text { o pobreza. } \\
\text { Normas sociales y culturas en } \\
\text { torno a la aceptación de los } \\
\text { castigos físicos. } \\
\text { No viviendas adecuadas y falta } \\
\text { de apoyo institucional al grupo } \\
\text { familiar. }\end{array}$ \\
\hline
\end{tabular}

Fuente: Organización Mundial de la Salud, Maltrato infantil - datos y cifras ${ }^{61}$.

En los casos de maltrato infantil propios del Síndrome del Niño Sacudido, la mayoría de las historias clínicas establecen que los factores desencadenantes son "los períodos de llanto inconsolable o episodios impredecibles en el niño, irritabilidad y rechazo al alimento; lo que genera frustración o angustia en el cuidador"62.

\subsection{Aspecto médico legal.}

La importancia del estudio de síndromes específicos de maltrato infantil, como el que nos ocupa, es orientada a determinar el grado de responsabilidad que recae sobre la persona que ha ejercido este tipo de acciones, lo que deberá abordarse a través de una intervención interdisciplinaria e interinstitucional: personal médico-forense, trabajadores sociales, psicólogos $\mathrm{y}$ funcionarios de la administración de justicia ${ }^{63}$.

adultos es el trabajo presentado por Alvaro Capano y Ayrel Pacheco, Estrés y violencia doméstica: un estudio en adultos referentes de niños, niñas y adolescentes, Prensa Médica Latinoamericana, Vol. VIII, 2014, pp. 31-42.

61 Se puede consultar la nota completa en la dirección electrónica https://www.who.int/es/news-room/fact-sheets/ detail/child-maltreatment

62 Arturo Loredo Abdalá, Jorge Trejo Hernández y otro, Síndrome del niño sacudido. Forma extrema de abuso físico infantil, Acta Pediátrica Mexicana, Vol. 32, 2011, 177.

63 Ibíd. 
En los casos del Síndrome del Niño Sacudido las lesiones pueden ser múltiples y de diversa índole, siendo el resultado fatal la muerte de la niña o niño. La anatomía patológica de este síndrome estará relacionada con las lesiones y secuelas que se le produzcan a la persona menor de edad, mismas que dependerán de la intensidad, su ubicación y la edad del infante ya que, lamentablemente, los casos de niños menores de 1 año son los que presentan lesiones más graves y la mayor letalidad ${ }^{64}$. Parte fundamental será el tratamiento de las secuelas y debe iniciarse muy precozmente. En muchas ocasiones las secuelas permanentes dependerán de la demora o la falta del tratamiento rehabilitador ${ }^{65}$.

Desarrollando un análisis jurídico penal, las lesiones provocadas a la persona menor de edad y propias de este síndrome pueden ser calificadas desde lesiones leves hasta gravísimas según como se tipifican en la legislación penal nacional. Sin embargo, en la mayoría de los casos podrá hablarse de una responsabilidad penal por producir lesiones gravísimas o graves ya que la mayoría de víctimas sufrirán secuelas de incapacidad importante ${ }^{66}$.

Con lo previamente acotado, hay que considerar lo manifestado por el doctor Franz Vega Zúñiga, quien menciona lo difícil que resulta para el profesional médico forense determinar el daño neurosensorial o neuropsicológico producto de traumas cráneo-cerebral. A su vez, indica que esta dificultad viene determinada "por lo delicado que es intentar objetivizar lo tremendamente subjetivo de los síntomas síquicos o sicológicos del paciente con 64 Enrique Paris Mancilla, Meneghello. Pediatría, Tomo I, 6ta. Edición, Buenos Aires: Editorial Médica Panamericana, 2013, 2601.

65 Giménez Pando y otros, 98.

66 Loredo Abdalá, 178. secuelas de trauma cráneo-encefálico (TCE)" $66^{67}$.

Vega Zuñiga, abordando el concepto y alcances del termino incapacidad, refiere que en el caso de traumas cráneo-encefálicos el perito forense deberá valorar el menoscabo por incapacidad fisiológica permanente producto del mismo. En consecuencia, citando a Mélennec, el doctor Vega indica que por Incapacidad fisiológica permanente se entenderá "la limitación de una o varias funciones orgánicas, intelectuales o psíquicas, con su corolario, la disminución parcial o total de las aptitudes en el terreno físico, intelectual o mental (comprender, pensar, formular juicios, etc.)"68.

Lo previamente acotado nos da una idea de que en estos casos la determinación de la responsabilidad penal no resulta tan simple. En primer lugar, hay que tomar en cuenta que no es hasta años después de seguimiento médico que se logran establecer otro tipo de afectaciones, por ejemplo, como perdida neuronal que produce comportamientos autistas. Incluso, los diagnósticos a largo plazo casi siempre establecen secuelas considerables como retraso mental, parálisis cerebral infantil y epilepsias, que no se diagnostican hasta mucho tiempo después ${ }^{69}$.

También es importante mencionar, respecto a la extensión de las obligaciones que tienen los padres de evitar que terceros ejerzan acciones de este tipo en contra de sus hijos, caso contrario, también podrían incurrir en responsabilidad penal ya que ostentan lo que se denomina "una posición

67 Franz Vega Zuñiga, Valoración médico legal de las secuelas neuro-sicológicas post trauma cráneo-encefálico, Medicina Legal de Costa Rica, Vol. 17 (2), 2001, pp. 27-36. Consultado a través de la dirección electrónica https://www.scielo.sa.cr/ $\underline{\text { scielo.php? } \text { pid }=\text { S1409-00152001000100007\&script }=\text { sci }}$ $\underline{\operatorname{arttext}}$

68 Ibíd

69 Rufo Campos, 42-43. 
de garante" que refiere a las obligaciones, entre otras, de alimentarlos, mantenerlos limpios, evitar lesiones físicas, etcétera ${ }^{70}$.

Sobre este punto, la Sala Tercera a través de Resolución no. 00869-2013 ha indicado

El ad quem sustentó su decisión al amparo de que no habían pruebas de que el endilgado maltratara a la menor (agresión activa), no obstante, dejaron de analizar un aspecto fundamental como lo era las múltiples y evidentes lesiones que presentaba la niña de cuatro meses (trauma cráneo encefálico con excoriación en la región parietal izquierda con infiltrado hemorrágico del cuero cabelludo en regiones frontal-parietal temporal y occipital derechas, en región parietal izquierda $\mathrm{y}$ fractura lineal parietal derecha, traumatismo torácico con fractura con callo óseo en el tercio posterior de la sétima costilla derecha y traumatismo en antebrazo izquierdo con facturadle radio), así como la actitud y el comportamiento que tuvo el imputado respecto de la niña que creía su hija biológica, claro está al amparo de la posición de garante que tenía, no solo de evitar las agresiones, sino de ejecutar una serie de acciones mínimas, a fin de preservar la vida e integridad de la menor $^{71}$.

70 Estamos ante los denominados delitos de "omisión impropia” establecidos en el artículo 18 del código penal. Según este tipo de delitos quien ostenta la posición de garante, en este caso los padres en relación a sus hijos, que responde por la omisión de la conducta mandada, imputándosele el no impedimento del resultado en virtud de su posición de garante. Así en Francisco Castillo Gonzáles, Derecho Penal Parte General, Tomo I, San José: Editorial Jurídica Continental, 2010, 330-332.

71 Sala Tercera de la Corte Suprema de Justicia, Resolución No. 00869-2013, del 12 de julio de 2013. Consultada el 10 de octubre de 2013 a través de la herramienta de búsqueda Nexus en la dirección electrónica https://nexuspj.poder-judicial. go.cr/document/sen-1-0006-778375

ISSN: 2521-5159 (En Línea)
El personal médico ante este tipo de casos clínicos esta en obligación de denunciar frente a las autoridades competentes los casos de maltrato en contra de personas menores de edad, tal como lo establece el artículo 49 del Código de la Niñez y la Adolescencia. Cuando se sospecha de un posible caso de maltrato infantil el diagnóstico que emite el o la médico, principalmente en las salas de emergencia, resulta de vital importancia para ilustrar al juzgador sobre la naturaleza de las lesiones, número, localización, posible mecanismo de producción y otros datos que se consideren pertinentes ${ }^{72}$.

En este aspecto, la Sala Tercera en relación a la obligación de denuncia del maltrato infantil establecida en el artículo 49 del Código de la Niñez y Adolescencia ha indicado

Dicha norma procura, esencialmente, resguardar a las personas menores de edad que puedan ser víctimas de abusos o maltratos y se encuentren en una situación particular tal que, de no ser por la denuncia del educador, $\boldsymbol{e l}$ médico o el funcionario, la conducta punible probablemente nunca sería denunciada. Se dirige, principal, aunque no exclusivamente, a proteger a los niños y adolescentes que sufran esas agresiones en el seno de su grupo familiar o comunitario y, por ende, el Estado promueve que sea el educador, el médico o el funcionario quien asuma un papel que, normalmente, le correspondería a los padres o representantes de la persona menor de edad, pero que estos no asumen por tener intereses contrapuestos, por ser los propios autores de la agresión o porque pretenden encubrir al autor ${ }^{73}$.

72 Calabuig, 321.

73 Sala Tercera de la Corte Suprema de Justicia, Resolución no. 00306 - 2008 de los 16 días de abril de 2020. Consultada a través de la herramienta de búsqueda Nexus el 29 de septiembre de 2020 en la dirección electrónica https://nexuspj.

Revista de Derecho. Vol. 42, No. 1, Año 2021 -33 
A su vez, la relevancia médico legal de este diagnóstico versará sobre el tipo de asistencia, tratamiento quirúrgico, continuidad de actos médicos, o de otra índole, necesarios sobre todo por las secuelas neurológicas que provocarán las lesiones, lo que resulta imperativo para determinar cuál es el supuesto legal en el que se enmarcan las acciones propias de maltrato infantil que ha ejecutado el agresor.

Las lesiones, según indica Javier Llobet Rodríguez, es un daño en el cuerpo o en la salud comprendiéndose en esta última tanto la salud física como mental ${ }^{74}$. Ahora bien, esta afectación puede ser de índole física o funcional, temporal o permanente y que puede ser cometida a título de dolo o culpa. indica que las lesiones. El código penal costarricense establece en sus artículos 123, 124 y 125 responsabilidad penal para lesiones gravísimas, graves y leves respectivamente.

En los casos de maltrato infantil propios del Síndrome del Niño Sacudido se ha establecido que las afectaciones a la salud de las personas menores de edad pueden llegar a afectar su capacidad normal de desarrollo por secuelas neurológicas principalmente, por ende, en la mayoría de casos en que pudiese probarse la responsabilidad de la persona acusada será por producir lesiones graves y gravísimas al niño o niña víctima.

Por ejemplo, en el caso de lesiones graves debe presentarse un debilitamiento persistente de la salud, es decir debe haber una disminución de la capacidad orgánica $\mathrm{y}$, como se establecía previamente, las secuelas que pueden sufrir las victimas del Síndrome del Niño Sacudido pueden ser desde la pérdida total o parcial de la vista, la capacidad de aprendiza y de habla e incluso presentar trastornos mentales que pueden poder-judicial.go.cr/document/sen-1-0034-407032

74 Javier Llobet Rodríguez,

34-|Revista de Derecho. Vol. 19, No. 1, Año 2021 determinarse como lesiones graves e incluso gravísimas dependiendo del tipo y la magnitud de la lesión provocada.

Cuando de las lesiones producidas por mecanismo de zarandeo resultare la muerte se estaría en presencia de lesiones denominadas mortales y que a su vez son una circunstancia agravante desde el enfoque legal al momento del análisis de reproche y la determinación de la pena. En ese sentido, y a modo ejemplificativo, el artículo 112 del Código Penal establece responsabilidad por homicidio calificado, cuya pena es mayor a la del tipo penal de homicidio simple, a quien mate "a su ascendientes, descendiente o cónyuge...", pudiendo también incurrir en responsabilidad por un homicidio calificado aquella persona que mate a otra que sea menor de doce años de edad.

\section{RECOMENDACIONES}

- Algunas recomendaciones que pueden aportarse para el personal médico, sobre todo cuando se tenga sospecha de que una persona menor de edad ha sido víctima de maltrato infantil físico propio del Síndrome del Niño Sacudido, son las siguientes:

- Es importante que el médico sospeche estar ante un caso de maltrato infantil cuando la historia contada por los padres no concuerde con los síntomas presentados por el paciente, sobre todo cuando se presentan consultas reiteradas por trauma, lesiones antiguas no tratadas o presentes en zonas corporales poco comunes.

- La interconsulta y el abordaje interdisciplinario son herramientas fundamentales de las que el médico que evalué a la víctima no debe prescindir, sobre 
todo cuando se considera que el menor ha sido víctima de lesiones propias del Síndrome del Niño Sacudido, ya que las lesiones pueden pasar desapercibidas y los síntomas pueden ser desde inespecíficos hasta el coma.

- Cuando se presente como resultado fatal la muerte de la víctima, no debe descartarse la realización de autopsias y estudios radiológicos en busca de lesiones esqueléticas ya que en el maltrato infantil son frecuentes sobre todo en las extremidades, el cráneo y las $\operatorname{costillas~}^{75}$. Y, como se pudo dilucidar de lo previamente anotado en torno al cuadro clínico del Síndrome del Niño Sacudido, los síntomas que permitirán orientar respecto al diagnóstico del mismo es la presencia de daño cráneo-encefálico.

- En todos los casos debe hacerse un diagnóstico diferencial: accidentes, alteración metabólica, usos y costumbres ${ }^{76}$. Esto porque muy rara vez podrá determinarse lo que en realidad sucedió a través del relato de los agresores.

\section{CONCLUSIONES}

- El maltrato infantil es un tema que debe importarnos a todos, principalmente por la dinámica y los ambientes cerrados a la luz pública en los que se desarrolla. Debemos ser conscientes de este flagelo ya que, si la denuncia de este tipo de violencia no es proporcionada por personal médico, personal docente o por algún otro familiar que se interese por la salud física y emocional de la persona menor de edad, hay una alta probabilidad de que se continúen ejerciendo este tipo de acciones en contra de la víctima.

75 Calabuig, 239. 321

76 Loredo Abdalá y otros, 179.
- El Síndrome del Niño Sacudido, al ser un tipo de maltrato de índole física es una de las formas más comunes de malos tratos ejercidos en contra de los niños y niñas menores de cuatro años. Sin embargo, es importante mencionar que es casi desconocido el mecanismo por el que puede provocarse, sobre todo por padres y madres primerizos.

- El diagnóstico del Síndrome del Niño Sacudido no es tarea fácil para el personal médico y de salud. En primer lugar, hay que considerar que los traumatismos craneales, infligidos por el mecanismo de zarandeo, pueden ir acompañados de fracturas óseas ocultas y a su vez hay que sumar la presencia de varias lesiones para desarrollar un diagnóstico de sospecha ${ }^{77}$. Es por esto que se debe realizar un abordaje interdisciplinario y realizar interconsultas.

- Los aportes medico legales en el abordaje del Síndrome del Niño Sacudido, y del maltrato infantil en general, estarán determinados por el detalle y claridad de la información que los médicos consignen en la historia clínica y en los diagnósticos, sobre todo, al momento de especificar el tipo de lesiones, cantidad, lugar del cuerpo donde se ubican, la antigüedad de las mismas y, en caso de ser posible, las secuelas que podrían presentarse.

- A su vez, y como indicaba la Organización Mundial de la Salud, no hay que olvidar que las acciones preventivas y socializadoras de temas como el maltrato infantil son de vital importancia ya que la protección de los niños, niñas y adolescentes en su condición de vulnerabilidad y sujetos a una figura de autoridad les posiciona en una situación de mayor riesgo.

77 Tejada Palacios y otros. 


\section{REFERENCIAS BIBLIOGRÁFICAS}

- ALVARAdo GUEVARA, Ana Teresa y Karen Garro Vargas. El síndrome del niño sacudido. Revista de Medicina Legal de Costa Rica, Vol. 25, 2008.

- BARRANTES ECHAVARRÍA, Rodrigo. Investigación: un camino al conocimiento, $2^{\mathrm{a}}$ ed. EUNED San José, 2016.

- CAlabuig, Gisbert. Medicina Legal y Toxicología, 6ta. Edición. Editorial Masson, 2004.

- CAPANO, Alvaro y Ayrel Pachecho. Estrés $Y$ Violencia Domestica: un estudio en adultos referentes de niños, niñas $y$ adolescentes. Prensa Medica Latinoamericana, Vol. VIII, 2014.

- CAstillo GONZÁles, Francisco. Derecho Penal Parte General, Tomo I. San José: Editorial Jurídica Continental, 2010.

- COHEN ROSENTOCK, Stephanie y Esteban Cob Guillén. Abuso sexual en el paciente pediátrico. Revista de Medicina Legal de Costa Rica, Vol. 36, 2019.

- EBERHARD, Ma. Eliana, Georgette Pose L. y otros. Maltrato infantil, TEC y diagnóstico por imágenes. Revista Chilena de Pediatría, Vol. 82 (3), 2011.

- GARCÍA MARTIN, Ruth y Benito Morentin. Valoración forense integral del maltrato infantil intrafamiliar. Revista Española de Medicina Legal, Vol. 46, 2020.
- LOREDO ABDALÁ, Arturo, Jorge Trejo Hernández y otro. Síndrome del niño sacudido. Forma extrema de abuso físico infantil. Acta Pediátrica Mexicana, Vol. 32, 2011.

- MADRIGAL, Édgar Alonso. Aspectos medico legales del síndrome del menor agredido. Acta Médica Costarricense, Vol. 52 (4), 2010.

- MANCILla, Enrique Paris. Meneghello. Pediatría, Tomo I, 6ta. Edición. Buenos Aires: Editorial Médica Panamericana, 2013.

- ORGANIZACIÓN DE LAS NACIONES UNIDAS. Convención sobre los Derechos del Niño, Resolución 44/25, 1989. Consultada a través de la página electrónica de la ONU a través de la dirección https://www.ohchr.org/ SP/ProfessionalInterest/Pages/CRC.aspx

- ORGANIZACIÓN MUNDIAL DE LA SALUD. Prevención del Maltrato Infantil: qué hacer, y cómo obtener evidencias, Francia, 2009.

- RÍOS, A., J. Casado Flores y otros. Maltrato infantil grave en la unidad de cuidados intensivos pediátricos. Anales de Pediatría, Vol. 71 (1), 2009.

- RUFO CAMPOS, M. El síndrome del niño sacudido. Cuadernos de Medicina Forense, No. 12, 2016.

- SÁNCHEZ, Juan Antonio y María Elena Albarrán. Medicina legal: concepto y definición. Historia. Importancia de la medicina legal. Relación con otras ciencias, así en Medicina Legal y Forense I, 2da. Edición. Valencia: Tirant lo Blanch, 2018. 
- TEJADA PALACIOS, Pilar, Noemí Núñez Enamorado y Berta Zamora Crespo. Traumatismo craneal no accidental. Boletín de la Sociedad Oftalmológica de Madrid, No. 54 (2), 2014. Consultada a través de la dirección electrónica_https:// sociedadoftalmologicademadrid.com/ revistas/revista-2014/m2014-03.html

- ULATE SOTO, Ileana / VARGAS MORÚA, Elizarda. Metodología para elaborar una tesisEUNED, San José 2016.

- VARGAS ALVARADO, Eduardo. Medicina Legal, 4ta. Edición. México: Editorial Trillas, 2012.

- VEGA ZUÑIGA, Franz. Valoración médico legal de las secuelas neuro-sicológicas post trauma cráneo-encefálico. Medicina Legal de Costa Rica, Vol. 17 (2), 2001.

- VÍQUEZ JIMÉNEZ, Mario Alberto. Maltrato físico en la niñez, un maltrato físico permitido. Estudio sobre autoridad parental. San José: Fondo de las Naciones Unidas para la Infancia, 2014.

\section{$\underline{\text { Legislación Nacional }}$}

- Asamblea Legislativa de la República de Costa Rica. Norma No. 7586 - Ley contra la Violencia Doméstica. San José, abril de 1996. Consultada el 22 de septiembre de 2020 a través de la herramienta de búsqueda SINALEVI en la dirección electrónica http://www.pgrweb.go.cr/scij/Busqueda/ Normativa/Normas/nrm_texto_completo. aspx?nValor $1=1 \&$ nValor $2=27926$
- Asamblea Legislativa de la República de Costa Rica. Norma No. 7739 - Código de la Niñez y la Adolescencia. San José, enero de 1998. Consultada el 22 de septiembre de 2020 a través de la herramienta de búsqueda SINALEVI en la dirección electrónicahttp://www.pgrweb.go.cr/ scij/busqueda/normativa/normas/nrm texto completo.aspx? param 1=NRTC\&p aram $2=1 \& n$ Valor $1=1 \& n$ Valor $2=43077 \& \mathrm{~s}$ $\underline{\text { trTipM }=\text { TC\&1Resultado }=4 \& \text { strSelect }=\text { sel }}$

\section{$\underline{\text { Jurisprudencia }}$}

- Sala Constitucional de la República de Costa Rica. Resolución No. 15751 - 2008, del 21 de octubre del año 2008. Consultada a través de la herramienta de búsqueda Nexus el 29 de septiembre de 2020 en la dirección electrónica https://nexuspj.poder-judicial. go.cr/document/sen-1-0007-421686

- Sala Tercera de la Corte Suprema de Justicia. Resolución no. 00306 - 2008 de los 16 días de abril de 2020. Consultada a través de la herramienta de búsqueda Nexus el 29 de septiembre de 2020 en la dirección electrónica https://nexuspj.poder-judicial. go.cr/document/sen-1-0034-407032

- Sala Tercera de la Corte Suprema de Costa Rica. Resolución No. 000716-2012 de los 20 días del mes de abril del año 2012. Consultada a través de la herramienta de búsqueda Nexus el 20 de septiembre de 2020 en la dirección electrónica https://nexuspj.poder-judicial. go.cr/document/sen-1-0006-781664 
- Sala Tercera de la Corte Suprema de Justicia. Resolución No. 00869-2013, del 12 de julio de 2013. Consultada el 10 de octubre de 2013 a través de la herramienta de búsqueda Nexus en la dirección electrónica https://nexuspj. poder-judicial.go.cr/document/sen-1-0006$\underline{778375}$

- Tribunal de Apelación de Sentencia Penal - Segundo Circuito Judicial de San José. Resolución 00591 - 2017 de los 19 días del mes de mayo del año 2017. Consultada a través de la herramienta de búsqueda Nexus el 29 de septiembre de 2020, en la dirección electrónica https://nexuspj.poder-judicial. go.cr/document/sen-1-0034-719509

- Tribunal de Apelación de Sentencia Penal de Guanacaste. Resolución No. 00335-2013 del 17 de diciembre de 2013. Consultada a través de la herramienta de búsqueda Nexus el 29 de septiembre de 2020, en la dirección electrónica https://nexuspj.poder-judicial. go.cr/document/sen-1-0034-595547

- Tribunal de Familia. Resolución No. 01624-2007 del 20 de noviembre de 2007. Consultada el 07 de octubre de 2020 a través de la herramienta de búsqueda Nexus en la dirección electrónica https://nexuspj.poderjudicial.go.cr/document/sen-1-0034-393051

- Tribunal de Familia. Resolución No. 003412001, del 05 de marzo de 2001. Consultada a través de la herramienta de búsqueda Nexus el 27 de septiembre de 2020 en la dirección electrónica https://nexuspj.poder-judicial. go.cr/document/sen-1-0034-144875

\section{$\underline{\text { Páginas electrónicas }}$}

- Biblioteca Nacional Virtual de Medicina de los Estados Unidos el 24 de septiembre de 2020 a través de la dirección electrónica https://medlineplus.gov/spanish/ency/ article/000713.htm

- Diccionario de la Real Academia Española. Consultado el 20 de septiembre de 2020, a través de la dirección electrónica https://dle. $\underline{\text { rae.es/cuadro }}$

- Observatorio de violencia de genero https:// observatoriodegenero.poder-judicial. go.cr/index.php/soy-especialista-y-busco/ estadisticas/delitos-sexuales

- Organización Mundial de la Salud, Maltrato Infantil: datos y cifras. Consultada el 23 de septiembre de 2020 en la dirección electrónica https://www.who.int/es/newsroom/fact-sheets/detail/child-maltreatment

- Organización Mundial de la Salud, Temas de Salud: maltrato de menores, consultado el 10 de septiembre de 2020, a través de la dirección https://www.who.int/topics/child abuse/es/

- "Traumatismo Cerebral: Esperanza en la investigación”, en la dirección electrónica https://espanol.ninds.nih.gov/trastornos/ el traumatismo cerebral.htm?css=print

- Se puede consultar la nota completa en la dirección electrónica https://www.who. int/es/news-room/fact-sheets/detail/childmaltreatment 